\title{
Medical Applications of Ultra-Short Pulse Lasers
}

\author{
J. E. Marion
}

B.-M. Kim

This paper was prepared for submittal to the Society of Photo Acoustic Engineers Bio-Laser '99

San Jose, California

January 21-26, 1999

June 8, 1999

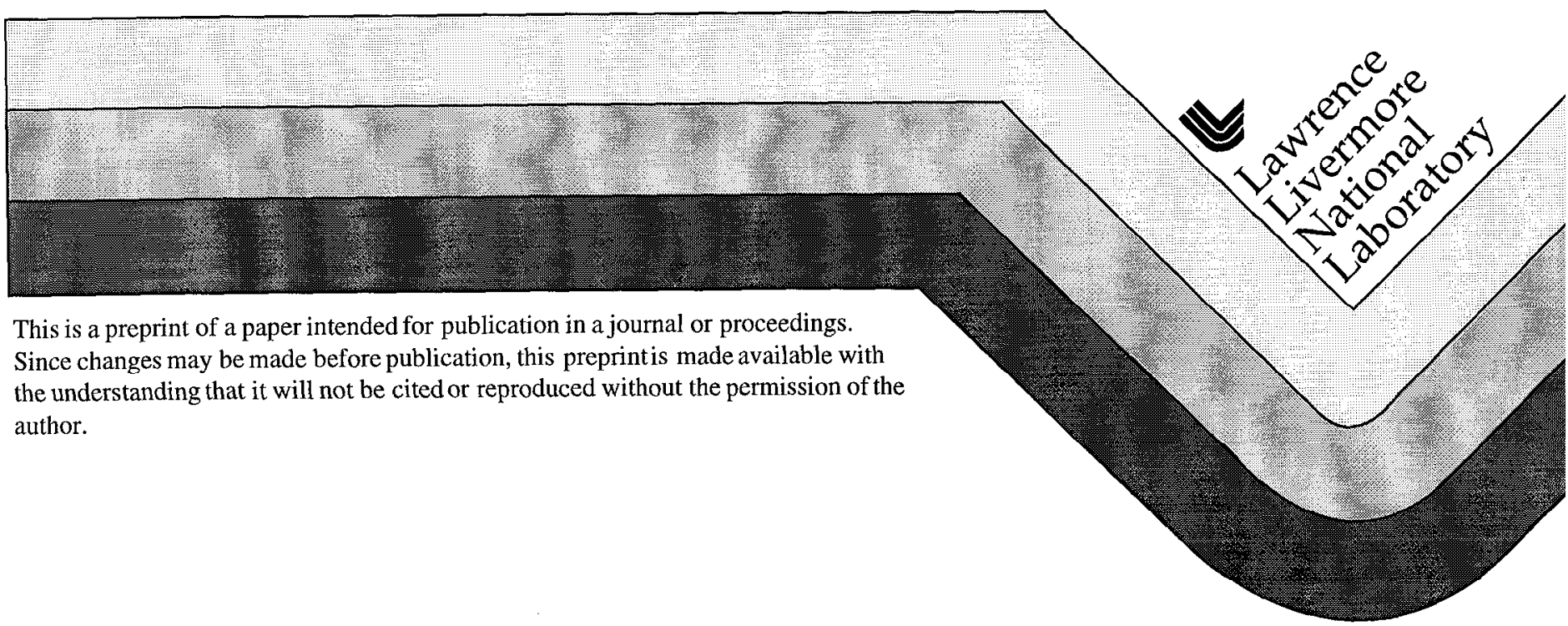




\section{DISCLAIMER}

This document was prepared as an account of work sponsored by an agency of the United States Government. Neither the United States Government nor the University of California nor any of their employees, makes any warranty, express or implied, or assumes any legal liability or responsibility for the accuracy, completeness, or usefulness of any information, apparatus, product, or process disclosed, or represents that its use would not infringe privately owned rights. Reference herein to any specific commercial product, process, or service by trade name, trademark, manufacturer, or otherwise, does not necessarily constitute or imply its endorsement, recommendation, or favoring by the United States Government or the University of California. The views and opinions of authors expressed herein do not necessarily state or reflect those of the United States Government or the University of California, and shall not be used for advertising or product endorsement purposes. 


\title{
Medical applications of ultra-short pulse lasers
}

\author{
John E. Marion II and Beop-Min Kim \\ Lawrence Livermore National Laboratory, L-399, P.O. Box 808, Livermore, CA 94551
}

\begin{abstract}
The medical applications for ultra short pulse lasers (USPLs) and their associated commercial potential are reviewed. Short pulse lasers offer the surgeon the possibility of precision cutting or disruption of tissue with virtually no thermal or mechanical damage to the surrounding areas. Therefore the USPL offers potential improvement to numerous existing medical procedures. Secondly, when USPLs are combined with advanced tissue diagnostics, there are possibilities for tissue-selective precision ablation that may allow for new surgeries that cannot at present be performed. Here we briefly review the advantages of short pulse lasers, examine the potential markets both from an investment community perspective, and from the view of the technology provider. Finally nominal performance and cost requirements for the lasers, delivery systems and diagnostics and the present state of development will be addressed.
\end{abstract}

\section{INTRODUCTION}

Ultra-short pulse lasers, having a pulse width of nominally 10-12 seconds or shorter, were originally develoned for spectroscopic purposes and dense plasma electrical conductivity measurements. ${ }^{1-3}$ As these short pulse laser sources became more accessible and more powerful, the unique ablation characteristics were noted. Chiefly, below about one ps, the physical mechanism for material removal ceases to be dominated by thermal processes and enters a region known as plasma-mediated ablation. The key point here is that below about one ps pulse width, material is ablated without appreciable thermal or mechanical damage to the surrounding tissue $e^{3}$.

There are a variety of intriguing materials processing applications that are being explored. These include machining of jet aircraft parts, nuclear weapons, high explosives and other applications. While these applications continuc to expand, its clcar that not all the good idcas have emergcd as yct and that a host of applications have yet to be discovered. This paper explores one specific application of Ultra short pulse lasers: medical applications.

There are several aspects to this market that merit discussion. Due to the complex nature of the USPL sources, and their current high cost, current applications are confined to relatively high value parts. Given the structure of the practice of medicine ablation of human tissue is a relatively low value. In order to become widely accepted in medicine, the cost of the laser sources must be consistent with this market. Secondly, the tolerance for unreliability or unpredictablity in the operation of the USPL goes from being annoying for the scientific market to being intolerable for the medical market. Thus sources must be reliable and predictable. Thirdly, the clinical setting is space-confined so the source must be both compact as well as semi-portable.

\section{ADVANTAGES OF ULTRA-SHORT PULSE I,ASERS}

In the ns regime, herein referred to as "long pulse" ablation is dominated by absorption of heat in the material. (Figure 1.) Material is heated through the boiling point to vaporization. As the material vaporizes, it is explosively ejected from the surface, leaving a jagged crater. In many materials, the high temperature in the surrounding material leads to thermal shock-related crack formation and to changes in the morphology of the surrounding areas (figure $2 \mathrm{a}, 3 \mathrm{a}$ ). In addition to the thermal affects, major changes can also result from the mechanical shock originating from the forceful ejection of material.

In contrast, below about one ps, an electron plasma forms at the surface and removes ions be electrostatic interactions (figure $2 b, 3 b$ ). This atom-by-atom removal does not involve substantial mechanical disruption 
to the surrounding areas. Further, as heat is the transport of energy over distance through phonon conduction, the thermal affects are minimal because time scale of the light pulse itself is on the same time scale as the ion-to-ion phonon transport. Therefore heating only occurs over an extremely local area.

The issue of pulse width scaling has been addressed.(0) Since its clear that sources with shorter pulse widths are likely to be more complicated the question arises, how short is the pulse width need to be in order to achieve the beneficial affects of Ultra-short pulse lasers. The general answer seems to be that below one ps pulses, the ablation is dominated by the plasma mediation. Above about $20 \mathrm{ps}$, thermal ablation dominates. In the intermediate areas, there may be some application specificity. For some applications, 5 or 10 ps pulses may be acceptable. For others, one ps, or less, may be required.

\section{THE MEDICAL APPLICATIONS}

Rather than simply listing an exhaustive set of potential medical applications, we've chosen to present representative medical uses, organized in a hierarchy based on the amount of material that must be ablated in a given surgery. This methodology was chosen to emphasize the important point that current commercial lasers only remove a small amount of material. So near term applications will clearly be those that only require a minimal volume of material removal. As more powerful sources become available, surgeries that require removal of a greater volume of material will become accessible.

TABLE 1 Potential Surgeries using USPLs

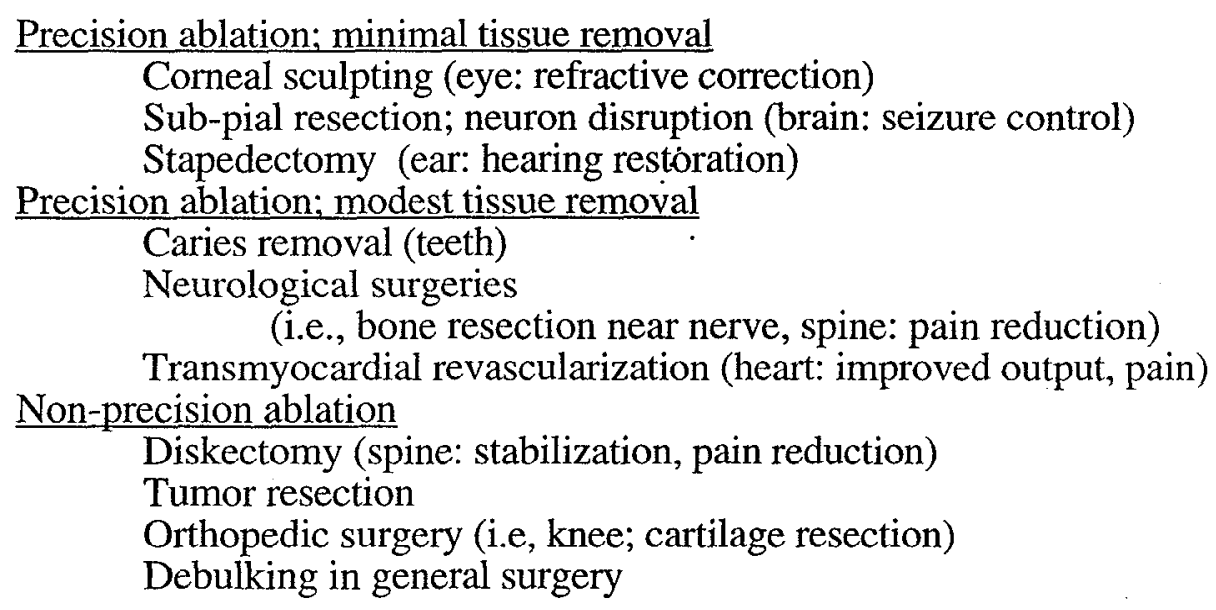

\subsection{Precision ablation with minimal tissue removal}

In table one we list the representative surgeries, grouped into three categories; those requiring minimal material removal, modest material removal and finally, even greater material volumes removed. In the first group, we list surgeries that take advantage of the precision ablation with no collateral thermal or mechanical damage, requiring on order of several hundred cubic microns of material removal. The first application is eye surgery for refractive correction. Pioneered by Intralase, Inc, () the surgery involves cutting a flap on the surface of the lens, removing a small slab of corneal tissue, and then restoring the flap over the surface. Very precise ablation allows the surgery to be very accurate. Combined with the improved predictability of the healing process allowed by the reduced thermal and mechanical damage to surrounding tissue, the USPL corneal sculpting procedure holds the promise of more accurate vision correction relative to current procedures. The next application is known as sub-pial resection. This epilepsy surgery is used for patients whose seizures are uncontrolled by drug therapies. The surgery involves the mechanical severing of neural connections around the focus of the seizures. Because of the morbidity associated with the damage caused by the surgery itself, this operation is annually performed on less than 1000 of the estimated 200,000 patients with epilepsy whose seizures are not controlled with drugs. By using short pulse lasers, with their minimal collateral damage profile, potentially this morbidity could be dramatically reduced. The 
third example of minimal tissue removal surgeries is stapedectomy. This is an ear surgery in which the stapes is removed and replaced with a prosthetic that connects the membrane covering the inner ear with the tiny bones that transmit the vibrations from the ear drum. Attachment of the prosthetic to these bones requires is technically challenging. If a small, 250 micron diameter hole could reliably be drilled in these bones, as with a USPL, the attachment would likely be more consistently successful. We have demonstrated the feasibility of this surgery on cadaver tissue.

Lasers to produce this minimal tissue ablation in time scales that are clinically acceptable would have to be on the order of one watt.

\subsection{Precision ablation with modest tissue removal}

Requiring a decade higher laser energy, perhaps $10 \mathrm{~mJ}$ per pulse at $1 \mathrm{kHz}$, the surgeries grouped as modest tissue removal range from caries removal to heart surgery. The dental application requires removal of a few cubic mm of material, and clearly, speed is important. We note that the lack of thermal and mechanical damage to surrounding tissue bodes well for both patient discomfort as well as safety from tooth nerve damage. Conceivably, this drilling might be accomplished without discomfort to the patient even without local anesthetization. Relative to other dental lasers, the USPL offers the advantage of ablating both hard and soft tissue with essentially equal efficacy.

The precision aspect of the USPL may prove advantagcous when used to resect bone or other tissuc from around sensitive structures such as nerves. A common surgery to relieve pain in the back and legs caused by degeneration of the intervertebral disk is the resection of bone adjacent to nerve at the laminae of the vertebra. This is technically challenging. USPLs offer the possibility of precise removal of the offending bone tissue without harm to the adjacent structures. Furthermore, diagnostics developed at LLNL () can differentiate between bone and other tissue and can be used to control the laser. The diagnostic collects the luminescent light that emanates from the electron plasma formed on the surface. This plasma luminescence forms in several tens of ns and with fast electronics can easily be collected and analyzed prior to the next pulse, one ms later. By controlling the laser output based on the signal form this diagnostic system, the laser can be turned off if the surgeon moves from cutting bone to cutting soft tissue. Effectively the bone is ablated but if the laser is pointed at soft tissue, the diagnostic turns off the laser before the next pulse.

Transmyocardial revascularization (TMR) is a therapy for end game heart patients with severe angina who are not candidates for by (further) pass surgery. It consists of laser drilling numerous small holes through the heart muscle (myocardium) into the ventricular chamber. While the exact causation mechanism is still being debated, TMR is clinically approved because it gives often dramatic decrease in angina. Various lasers have been used for TMR, including $\mathrm{CO} 2$, Holmium and Eximer lasers, with pulse widths ranging from ns to ms. Since the mechanism by which the pain relief is achieved is not yet agreed it has not possible to determine the optimal protile for the laser drilling with respect to thermal and shock conditions. Some preliminary results suggest that minimized scar tissue formation may be best, 0 there is some suggestion that USPLs, with their attendant minimal thermal and mechanical disruption to surrounding areas may be preferred.

Lasers to produce modest tissue ablation in time scales that are clinically acceptable would have to be on the order of ten watts.

\subsection{Precision ablation with modest tissue removal}

When high power USPLs become available there will be a class of no-precision ablation surgeries involving the removal of several cubic millimeters to centimeters of material that could be performed. Removal of the disk material from between vertebra in conjunction with spine stabilization is a common procedure. Use of USPLs in conjunction with clever packaging might make minimally invasive diskectomies much less technically challenging and substantially faster. A second surgery, tumor resection often requires even more material removal. Tissue type diagnostics might be applied to this surgery as well to differentiate between the tumor and the surrounding healthy tissue so that only the tumor cells are 
removed. Orthopedic surgeries often involve the removal of substantial mass. USPLs with their low collateral damage. Furthermore, tissue typing diagnostics would be helpful here as well for example to discriminate between cartilage which may need to be removed, and tendon which is not to be disturbed. General debulking in surgery requires an even higher level of power and may never end up being an appropriate use for USPLs.

\subsection{Non-ablation therapies}

Several therapies using USPLs are being pursued. Photogen, Inc. is using USPLs for two photon photodynamic therapy(). In photodynamic therapy an agent is administered to the patient that is preferentially absorbed by a particular tissue type, for example by a tumor. Using light of a specific color that is strongly absorbed by the photoactivator the tumor cells are disrupted. The two photon variant is potentially more selective. High fluence light of a color that has good penetration through tissue (red) is focused at the therapeutic site where it converts through a harmonic conversion process to a harmonic at shorter wavelength. The photoactivator is tuned to absorb at the shorter wavelength. The advantage is deeper penetration and better selectivity because the light is focused at a specific depth. Other USPL diagnostic guided therapies may be used.

\section{MEDICAL MARKETS}

\subsection{Patient Populations}

From an investor community view point the use of USPLs for medicine will depend on factor which include the size of the patient population. In general terms, investors are lonking for $\$ 100 \mathrm{M}$ markets. Using a scenario for commercialization of an USPL for a medical application in which each procedure used a disposable delivery device that was charged at $\$ 1000$, we would need a patient population of 100,000 to meet the $\$ 100 \mathrm{M}$ market requirement. Table 2 lists the annual patient loads for several candidate therapies. Many have case loads exceeding the 100,000 patient number. Also note that several of these therapies have a large "watchful waiting" population. The number of potential patients far exceed the number of surgeries because of efficacy, cost, side effects or other considerations. Disk surgeries and laminectomies are examples. Over $4 \mathrm{M}$ patients are admitted for hospitalization due to back pain, with less than $10 \%$ having surgery to resolve the problem. If a better treatment option were available, presumably a far greater number of these patients would seek surgery.

Table 2 Patient Populations for Potential USPLs Surgeries

\begin{tabular}{lc} 
Procedure & US Annual Cases (1996) \\
\hline Head, neck and skull surgeries & 212,000 \\
Facial bones and joints & 102,000 \\
Spinal Cord and spinal canal structures & 642,000 \\
Other cranial nerves & 69,000 \\
Disk surgeries*, Laminectomies* & 317,000 \\
Eye surgeries* & 283,000 \\
Ear surgeries & 68,000 \\
Epilepsy surgery* & 500 \\
Skin resurfacing* & 880,000
\end{tabular}

\subsection{Technology Markets}

The technologies that need to be developed for successful commercialization of USPLs include several aspects beyond the laser itself. The delivery system that takes the photons from the laser output to the general surgical area is important. The hand piece, microscope or other local delivery system would likely be customized for individual procedures. Lastly, diagnostics systems could potentially guide the surgeon to ablate only the targeted tissue. 
The laser system requirements, listed in table 3, illustrate near term and longer term needs. The laser itself is a capital item that may be an important barrier to commercialization. Present systems cost in excess of $\$ 100,000$. The present systems also do not meet the compactness or reliability needs of the clinical setting. Recent progress on small rugged oscillators bodes well for near term improvements in this area, though we note that the medical market is not the primary driver for these improvements. We further note that in our judgement, meeting the cost goals needed to penetrate the commercial medical field, will likely

. require more than clever packaging and economies of scale. That said, we believe that the design of USPLs

$\cdot$ is still in its infancy and that many of the best ideas have yet to come to the fore.

Table 3. Nominal USPL system requirements

\begin{tabular}{lll} 
& $\begin{array}{l}\text { Near term } \\
\text { (for clinical trials) }\end{array}$ & $\begin{array}{l}\text { Longer term } \\
\text { (for sales) }\end{array}$ \\
\hline power & few watts & $>10 \mathrm{~W}$ \\
pulse width & fixed; $<5 \mathrm{ps}$ & $\mathrm{fixed} ; 100 \mathrm{fs}-5 \mathrm{ps}$ \\
rep rate (nominal) & $\mathrm{kHz}$ & $\mathrm{kHz}$ \\
size & $2 \times 4 \times 4 \mathrm{feet}$ & $2 \times 2 \times 2$ \\
care and feeding & 220 or $110 \mathrm{~V}$ & 110 \\
air cooled & air cooled \\
reliability & "reasonably good" & $\begin{array}{l}\text { "scheduled } \\
\text { maintenance only" } \\
\text { cost targets }\end{array}$ \\
& $\$ 100 \mathrm{~K}-200 \mathrm{~K}$ & $\$ 30 \mathrm{~K}-150 \mathrm{~K}$
\end{tabular}

The delivery system is potential important as well. At the energy levels needed for reasonable tissue ablation rates the USPL pulses can not at present be transmitted within optical fibers because the intense USPL beam damages the fiber. Some ideas exist for use of fiber optic delivery, but success to date has been limited. Rather an articulated arm, or similar beam tube/mirror combination is typically used. This limits the applications somewhat, especially for catheter based procedures.

The local delivery system involving a hand piece or microscope is a critical element in the USPL surgical system. One key point is that the hand piece has the potential for being disposable. In the current managed care environment, investor favor a per procedure disposable because it simplifies the market, allowing the hospital to be reimbursed for a specific per procedure item with an identifiable cost. Clever designs of inexpensive disposable hand pieces might be central to successful commercialization of USPLs.

Diagnostics, which aid the surgeon by identifying specific tissue types, may also be central to successful application to medicine. Above we have described several candidate diagnostics, but the "wish list" of tissue type diagnostics is long. In addition to the plasma luminescence diagnostic, harmonic conversion of light in tissue has also been reported as a promising diagnostic (). Potentially, a suite of diagnostics might arm the surgeon with the best capability. We believe that perhaps the best diagnostics that take advantage of the short time scales and extreme fluences present during USPL ablation, have yet to be identified.

\section{CONCLUSIONS}

Although the USPL laser sources needed for medical applications are only now on the verge of being commercially available, the applications to medicine are compelling and numerous. We've presented several candidate applications in medicine which have patient populations which could support an investor community funded operation. To date, the refractive correction market is the only ablation therapy of which we are aware that is being addressed with USPLs. Two photon photodynamic therapy is a non-ablation market that is being addressed. As the lasers sources are commercially available in smaller, more rigged packages, and as the cost descends, more medical markets will be assessable. Promising diagnostics offer a key ingredient that may be central to the eventual wide spread use of USPLs in medicine.

This work was performed under the auspices of the U.S. DOE by LLNL under contract No. W-7405-Eng-48. 


\section{REFERENCES}

1. D. Strickland and G. Mourou, Opt. Commun. 56, 219 (1985)

2. A. Ng, et al, Phys. Rev, Lett. 57, 1595 (1986)

3. D.F. Price, et al, Phys. Rev. Lett. 75, 252 (1995)

4. 


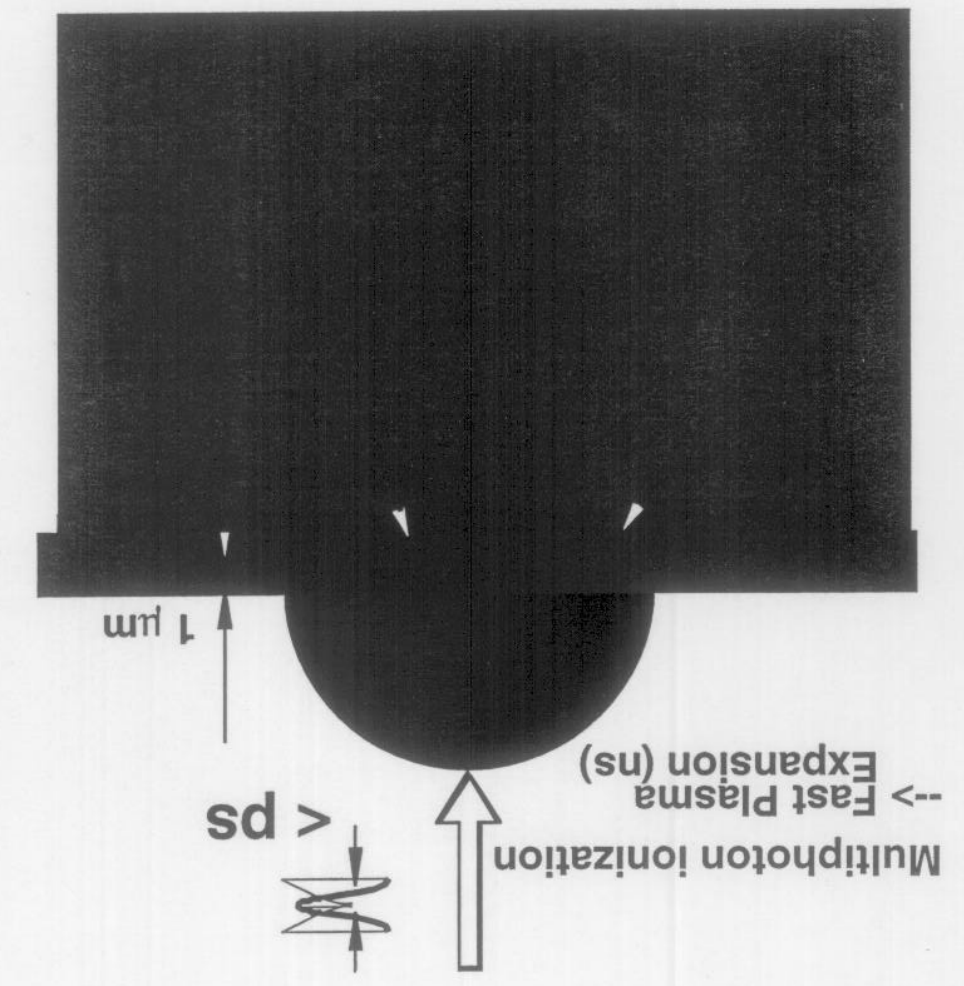

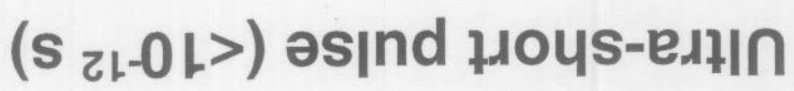

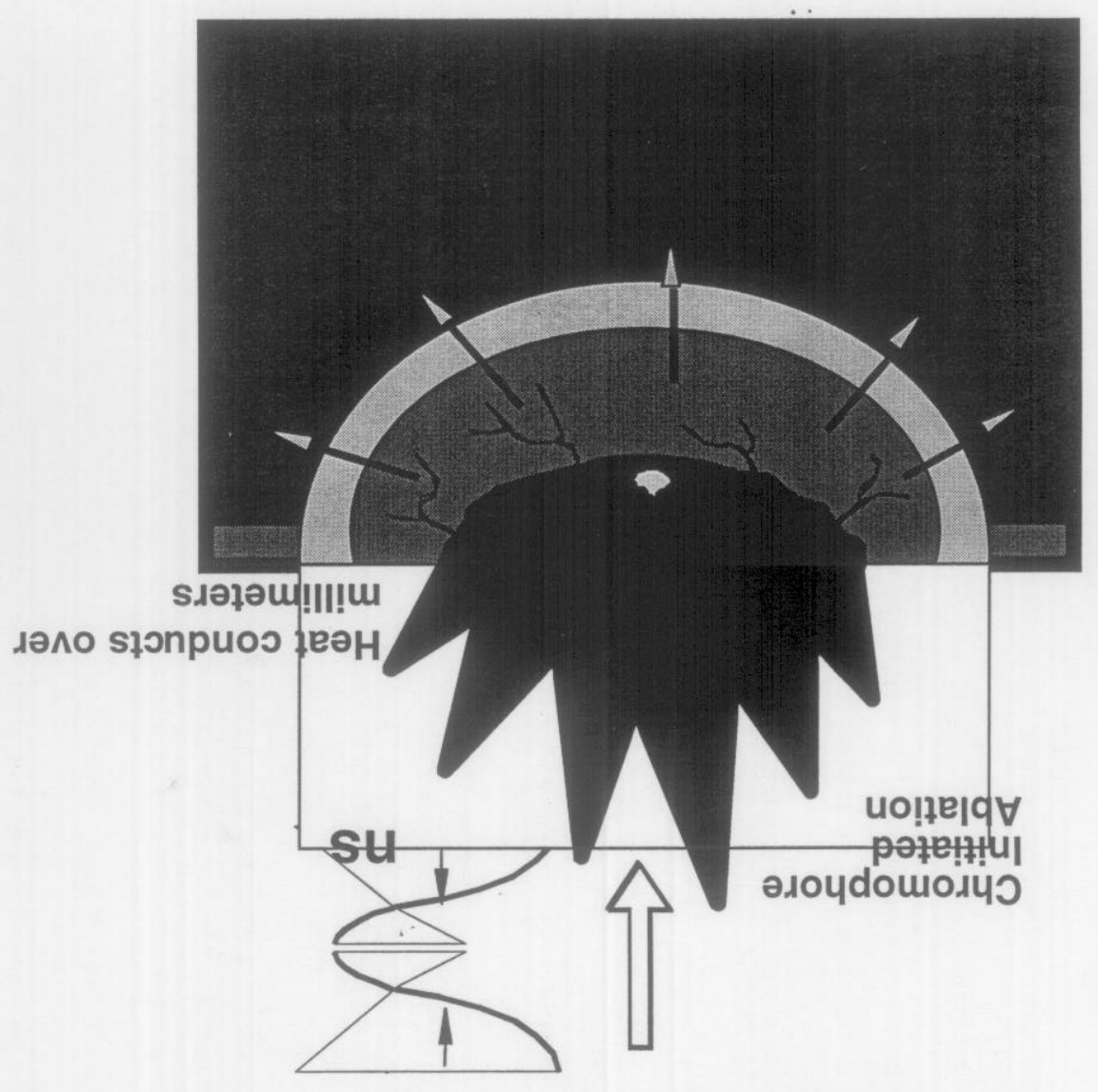

$\left(s_{6-0 L}<\right)$ əs|nd 6uo7 

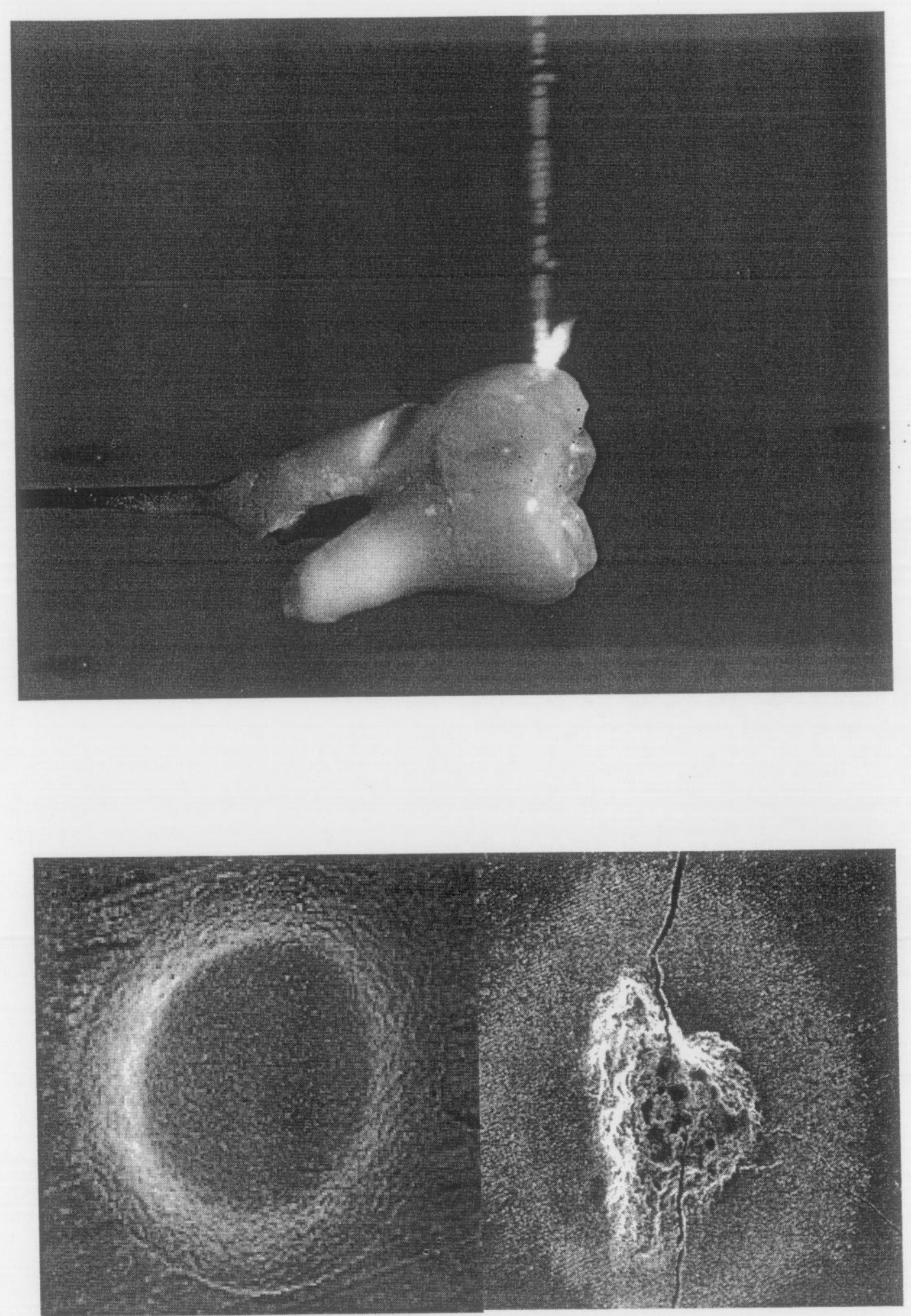

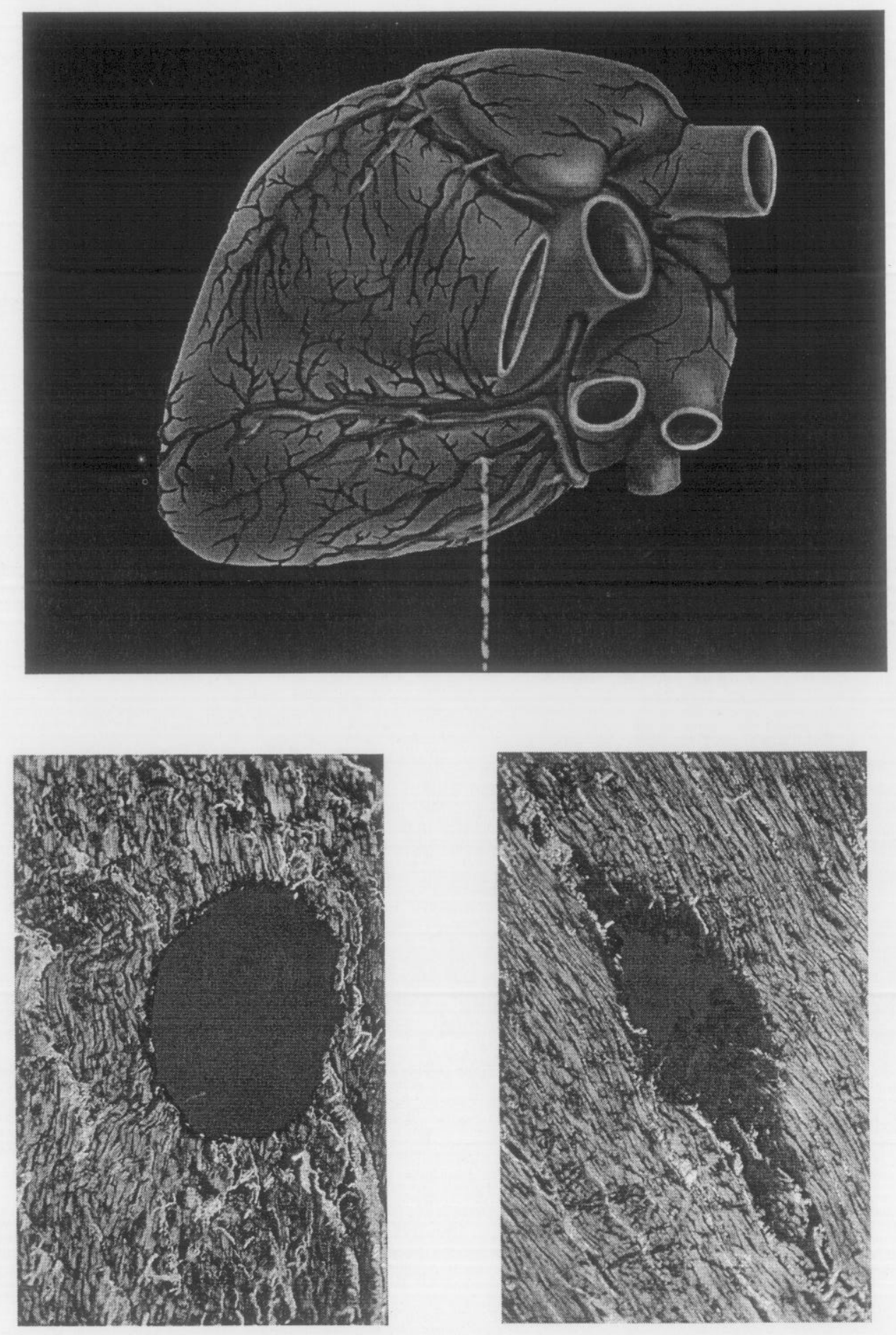